\title{
Methodology for Prior Evaluation of Interoperability
}

\author{
Mamadou Camara, Yves Ducq, and Remy Dupas \\ University of Bordeaux, IMS, CNRS 5218 \\ 351 cours de la Libération, 33405 Talence cedex, France \\ \{mamadou. camara, yves. ducq, remy. dupas\} @ims-bordeaux. fr
}

\begin{abstract}
Interoperability is the ability of two or more systems or components to exchange information and use the exchanged information without special effort on either system. This paper attempts to propose a framework for evaluating and improving interoperability in each one of partners collaborating in a supply chain. The definition of the framework is based on three important hypotheses. The first hypothesis supposes the existence of interoperation activities which correspond with the part of business processes representing efforts for interoperability. The second one is the definition of Key Performance Indicators (KPIs) as aggregation of time, cost and quality performance for interoperation activities and also for actual business activities. The third one proposes to analyze impact of interoperability investments on enterprise objectives in two steps: the first step is the evaluation of the impact of interoperability solutions in KPIs; the second step analyzes the impact of improving KPIs in the achievement of enterprise's operational, tactical and strategic objectives. A methodology for prior evaluation of interoperability and improvement based on this framework is also proposed. The goal of this methodology is the validation of interoperability solutions to be implemented.
\end{abstract}

Keywords: Enterprise Interoperability, Interoperability Measurement, Business Process Modeling, Business Activity Monitoring, Business Process Simulation.

\section{Introduction}

Interoperability is the ability of two or more systems or components to exchange information and use the exchanged information without special effort on either system [1]. Approaches developed in the literature to measure and improve interoperability can be categorized into qualitative and quantitative models. Qualitative models propose interoperability evaluation methods based on maturity models. Quantitative models use indicators to measure interoperability characteristics like time, cost and quality of interoperation. The main limitation of these approaches is that they are partial because the considered interoperability is disconnected from enterprise objectives. In this study, the goal is to propose a quantitative methodology for interoperability evaluation and improvement. For this, it is necessary to evaluate how interoperability investments participate to the achievement of enterprise objectives. A framework is proposed as a foundation to address this issue. This framework is based on the physical and the decisional systems of the GRAI conceptual reference model [2]. The framework is composed of three complementary and connected layers: the 
interoperability investments layer, the operational interoperability impact layer, and the tactical and strategic interoperability impact layer.

The paper is structured in six sections. Section two presents the state of the art of Performance Measurement System and Causal Performance Measurement Models. Section three introduces interoperability measurement methods existing in the literature. Section four describes framework. The structured methodology is described step by step in the fifth section. To finish, a short example is given in section six.

\section{Performance Measurement System and Causal Performance Measurement Models}

To evaluate interoperability we must connect it with enterprise objectives. For that purpose, we need, in the one hand, a Performance Measurement System to define performance indicators. In the other hand, we need a Causal Performance Measurement Models to analyze causation between indicators in the Performance Measurement System. A performance measurement system is a set of strategic objectives and performance metrics applied throughout the entire enterprise [3]. If we consider the literature, there are a lot of methods to define performance indicators or more generally performance measurement system starting from enterprise strategy or from customer satisfaction. Balanced Scorecard [4] or the Performance Prism [5] or QMPMS [6] define the indicators focusing only to the strategic level. The definition of objectives in coherence with performance indicators and decision variables, and its extension to all levels are the main reasons why we've chosen to work with ECOGRAI. According to Kasperskaya [7], causal performance measurement models were substituted for traditional performance measurement systems, because they enable not only to measure and control but, also guide the companies' performance. Balanced Scorecard strategy map [8], Action-profit linkage (APL) [9] and the graph of decomposition [10] belongs to the most important existing causal performance measurement models. For the methodology for interoperability evaluation a causal performance measurement model will be defined using elements from these three models.

\section{Interoperability Measurement}

This section presents the literature of qualitative and quantitative measurement of interoperability. The INTEROP framework is used first to give some basic definition of concepts related to interoperability. It provides three explicitly defined interoperability dimensions to allow defining interoperability: interoperability barriers, interoperability concerns and interoperability approaches [11]. Barriers are incompatibilities of various kinds and at various enterprise levels. The incompatibilities obstruct the sharing of information and prevent from exchanging services. Interoperability concerns represent the various enterprise levels where interoperations can take place: Data, Services, Process and Business [11]. Interoperability approaches are basic ways to remove barriers: integrated approach, unified approach and federated approach [11].Solutions for interoperability remove barriers at a particular enterprise level (concerns), through a specific interoperability approach [12]. The solutions are then respectively related to the three dimensions. 
Interoperability models that have proposed interoperability measures can be classified into qualitative and quantitative models. Mostly, qualitative models are designed as a mean of attaching a label or maturity level to a specific type of interoperability [13]. Each maturity level of a qualitative model is equivalent to an interoperability sophistication degree [14]. Levels of Conceptual Interoperability Model [14], System of Systems Interoperability (SoSI) Model [15] and Organizational Interoperability Agility Models (OIAM) [16] can be cited as qualitative models based on maturity levels.

Quantitative models are approaches that have numerical measures of interoperability characteristics [17], [13], [18], [19], [20], [21]. The measurement of interoperability is done in operational phase of the collaboration between two enterprises and allows them quantifying their interoperability and being able to improve it. Some equivalence can be noted between interoperability measures defined in the literature:

- Quality of exchange [19] and connectivity [17].

- Time of interoperation [19], data latency [17] and cycle time [20]

- Reliability [20] and conformity [19]

In our opinion, time, cost and quality are limited number of measures which encompasses all other types of interoperability efforts. Measuring interoperability with these three characteristics is an interesting option that we want to develop in this study.

\section{Interoperability Evaluation Framework}

This work proposes a framework for evaluating interoperability in each one of partners collaborating in a supply chain (Fig. 1). The framework should enable an understanding of how interoperability influences the achievement of enterprise objectives. The framework is partly inspired by the Lebreton, et al.'s [20] IIAM, but it is organized according to the GRAI conceptual reference model. The framework is composed of three layers: the interoperability investments layer, the direct (operational) impact layer and the tactical and strategic impact layer. The interoperability investments layer is aimed to study relations between elements located in the structural level of the physical system of the GRAI conceptual reference model. Theses elements are interoperability barriers, solutions for interoperability, internal or collaboration business processes and the business and interoperation activities they contain (Fig. 1). The main hypothesis of our model is that business processes can be broken up into business activities and interoperation activities. Business activities are those which create value in business process. Interoperation activities are non-value-added activities representing efforts for the interoperability of information exchanges between partners. Solutions for interoperability eliminate interoperation activities by deleting interoperability barriers. This is the way internal or collaboration business processes are transformed by solutions for interoperability.

The direct (or operation) interoperability impact layer is composed of two sub-layers. Its first sub-layer measures the interoperability impact on the operational level of the physical system of the GRAI conceptual reference model. This sub-layer contains business indicators, interoperation indicators and key performance indicators. Interoperation indicators are defined on interoperation activities and business indicators defined on 
business activities. Key performance indicators (KPI) are considered as aggregations of business and interoperation indicators of all activities of a business process in order to measure the performance of the latter. In the general case, this aggregation corresponds to the summation of indicators values of all activities. Interoperation indicators, business indicators and key performance indicators (KPI) are all of three types: Average Elapsed Duration, Average Cost and Percentage of Failure. These measures are first quantifications of the operational impact of interoperability investments on business processes in terms of performance. The second sub-layer is composed of decision centers of the operational level of the decisional system of GRAI conceptual reference model. It enables to measure the impact of interoperability in terms of achievement of objectives of the operational level. Some operational objectives are directly related to process key indicators while the others only indirectly.

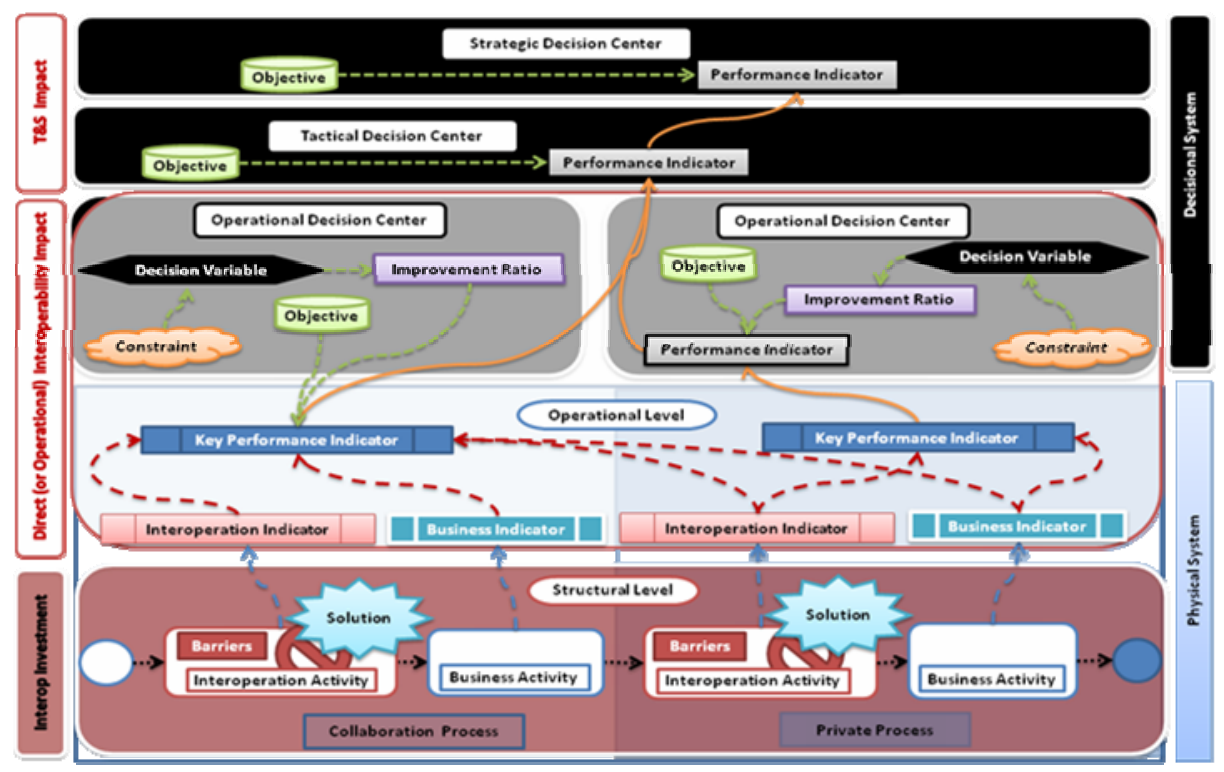

Fig. 1. Interoperability Impact Evaluation Framework

In the first category, the objectives are measured by operational performance indicators that are equivalent to key performance indicators because they are defined as process cost, time or quality (left side Fig. 1). In the second case, performance indicators are not defined as measures of time, cost and quality of business processes (right side Fig. 1). They can be related for example to equipments used or peoples working on physical level. Objectives are not directly related to key performance indicators in this situation. The tactical and strategic impact layer uses the tactical and strategic performance indicators of the GRAI decisional system to evaluate impact of interoperability investments on high level objectives (Fig. 1). The framework is founded upon a performance measurement system and a causal performance measurement model. Indicators in the performance measurement system are grouped in four components: process 
KPIs, operational performance indicators (O_PI), tactical performance indicators (T_PI) and strategic performance indicators (S_PI).The causal performance measurement model links together indicators of the four components, and materializes relation between the layers of our framework. It establishes how interoperability impact at operational level translates into benefits at tactical and strategic levels.

\section{Methodology for Interoperability Evaluation and Improvement}

The methodology that we propose aims to evaluate the interoperability and its impact in a supply chain. It is based on the interoperability evaluation framework. The methodology provides prior evaluation of interoperability in order to validate solutions for interoperability to be implemented on the basis of their capacity to improve the achievement of enterprise objectives. This prior evaluation takes place during "As-is" and "To-be" situation of the project. "As-is" situation describes how activities (physical system) and decisions (decisional system) are actually performed in the supply chain. "To-be" situation represents the transformations of the "As-is" situation expected as a result of the implementation of solutions for interoperability. Steps in the methodology can be grouped in three blocks: Learning, Design and Validation. The learning builds a causal performance measurement model linking together process KPIs to performance indicators of the different levels in the decisional system (Fig. 2.). As all the variables of our causal performance measurement model are continuous, the task of predicting performance indicators from process KPIs is a numerical prediction. Our model is built with regression analysis because, according to Han, et al. [22 p. 24] it is the most often used statistical methodology for numerical prediction. The learning block builds also the

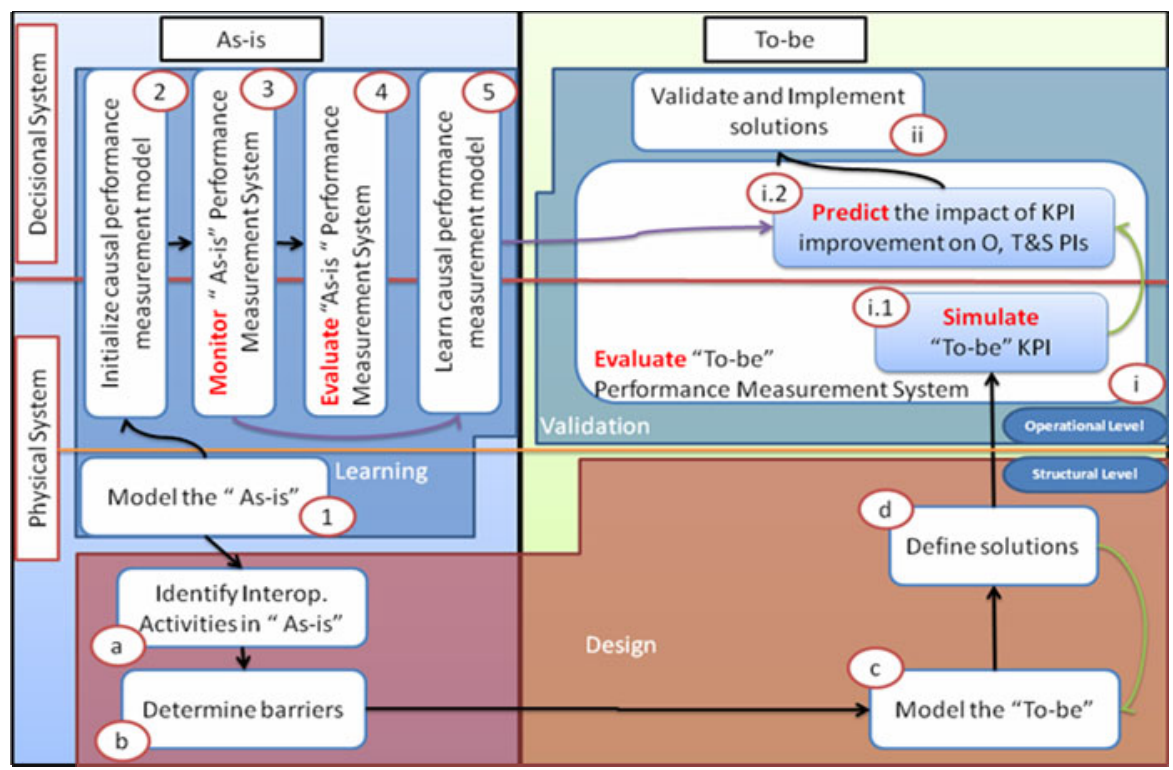

Fig. 2. Interoperability impact evaluation 
"As-is" performance measurement system which represents averages of measures collected in the "As-is" situation. The Design block is aimed to find transformations needed in physical and decisional systems to solve problems related to interoperability (Fig. 2.). The validation block is aimed to validate solutions for interoperability by evaluating their improvement capacity. The first task is the evaluation of the "To-be" performance measurement system which is released in two stages (i Fig. 2.).

First the "To-be" KPIs are estimated a prior by simulation of the "To-be" process model (i.1 Fig. 2.). The impact of the KPI improvement on operational, tactical and strategic performance indicators is predicted using the causal performance measurement model built in the learning block (i.2 Fig. 2.). The comparison between the "Asis" and the "To-be" performance measurement systems represents the improvement capacity. The validation of solutions for interoperability is the second task of this block (ii Fig. 2.) and it is based on the results of this comparison.

\section{Application of the Methodology}

The example used to illustrate the applicability of our methodology is about a supply chain where an interoperability investment is undertaken to improve the quality of the collaboration. Partners involved in this collaboration are: the customer (an ecommerce company), the stockist, the customs declarant and the customs. The first element of our example is a "As-is" goods entry (collaboration) process in which all the four partners participate. To get the "To-be" goods entry process model, it suffices to take the model of "As-is" process and remove its interoperation activities. Influence relations between the variables of the causal performance measurement model were established by managers. The process KPIs component of the Performance Measurement System contains only the Computerization time measure. The Computerization time represents the delay between the moment when the customer places his order for goods entry and the moment when the Stockist informs him that goods entry is closed and the Material Accounting is updated. It is in the same time the Average Elapsed Duration of the goods entry process and an operational performance indicator for the objective "satisfy demand" of the goods entry decision center. The customers can run the goods output process and sell goods only once the goods are registered in the system. For this reason managers hypothesize that there is a causal relationship between Computerization time variable (process KPIs), and number of outputs of pallets per month (O_PI), number of outputs of boxes per month (O_PI) and number of outputs of units per month (O_PI). The Handling Turnover (T_PI) receives influence from theses three operational performance indicators. An improvement in Handling Turnover will drive an increase Handling Turnover in relation to staff (T_PI) and Sales turnover (T_PI). Both the Price of logistic services (S_PI) and Total turnover compared to department cost (S_PI) are impacted by the Handling Turnover in relation to staff (S_PI). The Sales turnover indicator belongs to the customer while others belong to the stockist.

\section{Conclusion}

This study aimed to provide the means for evaluating the interoperability and its impact on enterprises in a supply chain. We have proposed an analytical framework that 
introduces concepts related to interoperability and establishes their relations. The core element of this framework is the causal relationship between process key performance indicators and the objectives of partners, at all decisional levels. A structured methodology has been also proposed to perform the priori evaluation of interoperability. This methodology is organized around the validation and implementation solutions for interoperability. The use of business process models to locate interoperation activities and interoperability barriers and to measure performance is an important contribution of the methodology. That enables quantifying interoperation indicators with existing simulation and monitoring tools used in business process management.

In future work, the methodology can be completed with a posterior evaluation which would take place once improved business processes have been implemented using solutions for interoperability. Posterior evaluation would build "Implemented" Performance Measurement System and compare it with the one of the "As-is" situation in order to measure real benefits of interoperability investments.

\section{Acknowledgement}

This work was done in the frame of ASICOM (Architecture de Système d'information Interopérable pour les industries du Commerce), a project labeled by the "Pole de Compétitivité" PICOM of the region Nord-Pas-de Calais (Lille) in France and funded by the FUI (Fonds Unifie Interministériel). The Goal of the ASICOM project is to allow e-commerce companies to be connected at low cost, in short time and with flexibility to their partners and to the custom administration, using an interoperable information system. Examples used to illustrate the applicability of our methodology are taken from this project.

\section{References}

1. Access, Committee on Cataloging: Description \&. Task Force on Metadata (2000)

2. Doumeingts, Guy. The GRAI Methodology (2004)

3. ManageMentor, Harvard. Performance Measurement Systems, http : / /www . sema . org/. [Online] 1.0.1120.9999 1.0.1 .5/16/2007 12:17:49 PM (2007), http://www.sema.org/hmm10/performance_measurement/what_is_a_ performance_measurement_system.html

4. Kaplan, R.S., Norton, D.P.: The Balanced Scorecard: Translating Strategy Into Action. Harvard Business School Press, Boston (1996)

5. Neely, A., Adams, C., Kennerley, M.: The Performance Prism: The Scorecard for Measuring and Managing Business Success. Edition Prentice Hall (2002)

6. Bititci, U.S., Suwignjo, P., Carrie, A.S.: Strategy management through quantitative modelling of performance measurement systems. International Journal of Production Economics 69 (2001)

7. Kasperskaya, Y.: Essays on Causal Performance Measurement Models. Universitat Pompeu Fabra (2007)

8. Kaplan, R.S., Norton, D.P.: Strategy maps: converting intangible assets into tangible outcomes. Harvard Business Press, Boston (2004)

9. Epstein, M.J., Westbrook, R.A.: Linking Actions to Profits in Strategic Decision Making. Mit Sloan Management Review (2001) 
10. Ducq, Y., Vallespir, B., Doumeingts, G.: Coherence analysis methods for production systems by performance aggregation. Int. J. Production Economics (2001)

11. Chen, D., Doumeingts, G., Vernadat, F.: Architectures for enterprise integration and interoperability: Past, present and future. Computers in Industry (2008A)

12. Naudet, Y., et al.: Towards a systemic formalisation of interoperability. s.l.: Computers in Industry (2010)

13. Ford, T., et al.: The Interoperability Score. In: CSER 2007 (2007)

14. Tolk, A., Muguira, J.: The Levels of Conceptual Interoperability Model. In: The 2003 Fall Simulation Interoperability Workshop (2003)

15. Morris, E., et al.: System of Systems Interoperability (SOSI): Final Report. The Software Engineering Institute (2004)

16. Kingston, G., Fewell, S., Richer, W.: An Organizational Interoperability Agility Model. In: The 10th Command and Control Research and Technology Symposium, CCRTS (2005)

17. Kasunic, M., Anderson, W.: Measuring Systems Interoperability: Challenges and Opportunities. Software Engineering Measurement and Analysis Initiative (2004)

18. Ford, T., et al.: A Survey on Interoperability Measurement. In: 12th International Command and Control Research and Technology Symposium (2007B)

19. Chen, D., Vallespir, B., Daclin, N.: An Approach for Enterprise Interoperability Measurement. In: MoDISE EUS 2008 (2008B)

20. Lebreton, B., Legner, C.: Interoperability Impact Assessment Model: An Overview. In: Enterprise Interoperability II: New Challenges and Approaches, pp. 725-728. Springer, London (2007)

21. Grandin-Dubost, M., et al.: Using IIAM to Assess Interoperability Investments: A Case Study. In: Enterprise Interoperability II. New Challenges and Approaches, pp. 119-123. Springer, London (2007)

22. Han, J., Kamber, M.: Data Mining: Concepts and Techniques. Morgan Kaufmann Series in Data Management Systems (2001) 